\title{
Statistical Investigation of Physical and Geometrical Parameters in Close Binaries using the ASAS Database
}

\author{
J. Nedoroščik ${ }^{1}$, M. Vaňko ${ }^{2}$ and Š. Parimucha ${ }^{1}$ \\ ${ }^{1}$ Institute of Physics, University of P.J. Šafárik, Košice, Slovakia \\ email: prokyon87@gmail.com \\ ${ }^{2}$ Astronomical Institute of the Slovak Academy of Sciences, Tatranská Lomnica, Slovakia \\ email: vanko@ta3.sk
}

\begin{abstract}
The main goal of this work was to find dependencies between Fourier coefficients, which were developed by light curve fitting with Fourier polynomials. The light curves were acquired from the ASAS database (All Sky Automated Survey). In this statistical research it was necessary to sort and modify these data, because light curves of eclipsing binaries are just part of a bigger database, which contains the light curves of pulsating variable stars, novas etc. It was required to phase and normalize all of our light curves, that it could be possible to use a program to fit light curves with Fourier coefficients. Thereafter, we were looking for relations between Fourier coefficients.
\end{abstract}

Keywords. photometry, CCD

\section{Introduction}

The ASAS† database contains about 11240 eclipsing binaries or candidates for eclipsing binaries. Light curves of these systems have been phased and normalized, that we can use program, which expands light curves to polynomial according to Fourier coefficients. In order to find Fourier coefficients we have used the following expression:

$$
S(x)=a_{0} / 2+\sum_{n=1}^{N}\left[a_{n} \cos (n x)+b_{n} \sin (n x)\right]
$$

For all light curves we obtained file with eleven Fourier coefficients.

\section{Results}

Some of these Fourier coefficients represent directly physical or geometrical parameters Hambalek (2006). We were interested in the relation between $a_{2}$ and $a_{4}$. Thereafter, we were looking for boundary which determines detached systems from semi-detached and contact systems for synthetic light curves (see Fig. 1, right) and light curves for real systems (see Fig. 1, left). Selam (2004) published his equation for this boundary as follows:

$$
a_{4}=a_{2}\left(0.125-a_{2}\right)
$$

$\dagger$ http://www.astrouw.edu.pl/ 

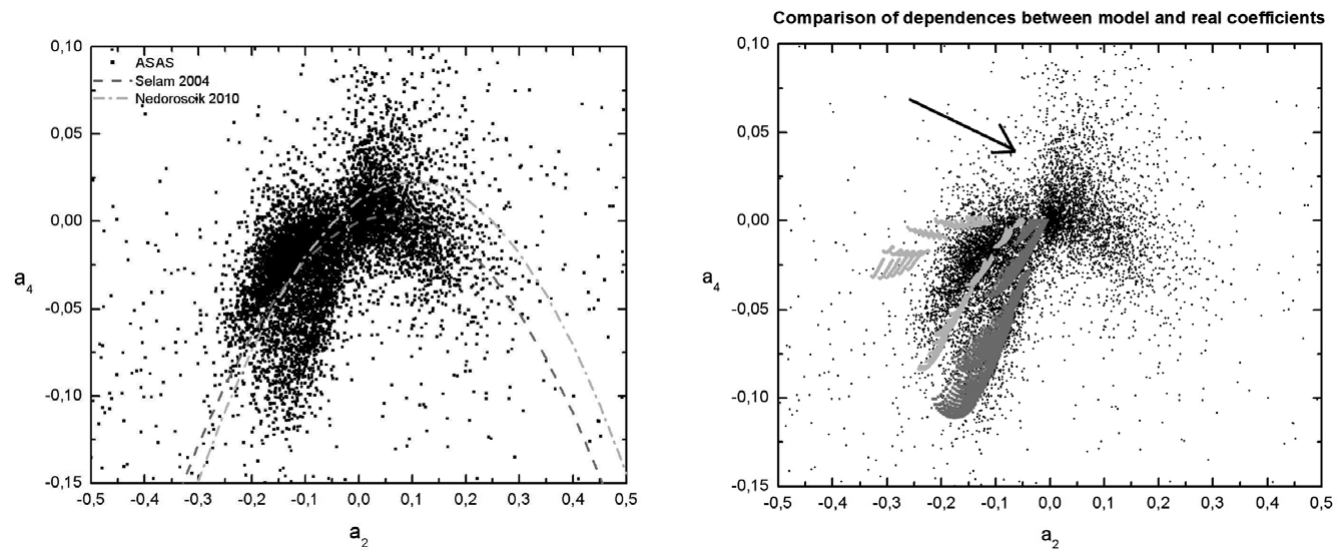

Figure 1. Left Boundary determination between EA types and EB, EW types. The plot represents real systems from ASAS database. Right Comparison of fourier coefficients relation for real (points) and model (lines) light curves.

This equation was not correct for our model light curves we needed to derive own boundary:

$$
a_{4}=-1.06011 a_{2}^{2}+0.21635 a_{2}+0.01229
$$

By these boundaries we can determine types of eclipsing binaries. When we compare dependences of these two Fourier coefficients for model and real light curves (see Fig. 1), we can estimate some physical (e.g. mass ratio) or geometrical (e.g. inclination) parameters. It is necessary to model light curves with high precision to obtain correct estimation of physical and geometrical parameters of binary systems. Interesting is the area marked by arrow (Fig. 1, right). We do not know, what kind of binaries represents this area. These stars could be close binaries with a lot of spots, pulsating stars or it could be systems with exoplanets.

\section{Acknowledgements}

This work has been supported by project VEGA 2/0094/11

\section{References}

Hambalek, L. 2006, Diploma thesis

Selam, S. O. 2004, A\&A, 416, 1097 